\title{
Description of non-diffusive solar cosmic ray propagation in a homogeneous regular magnetic field
}

\author{
Yu. I. Fedorov and B. A. Shakhov
}

\begin{abstract}
Main Astronomical Observatory National Academy of Sciences of Ukraine and Isaac Newton Institute of Chile, Kiev Branch, 03680, Zabolotnoho 27, Kiev, Ukraine

Received 1 August 2002 / Accepted 29 November 2002

Abstract. In the present paper the propagation of energetic charged particles in a magnetic field with a homogeneous regular component is studied. On the basis of the Boltzmann equation the analytical expressions for particle density and anisotropy are derived under instantaneous isotropic injection of particles into the scattering medium. Starting from the set of equations for spherical harmonics of the distribution function the new transport equation taking into account the second harmonic is carried out. The solution of this transport equation is reached and comparison with analytical solutions of the kinetic equation is performed. The telegraph equations for particle density and flux are derived and their solutions are analyzed. The transport of energetic particles under multiple small angle scattering is considered.
\end{abstract}

Key words. diffusion - radiative transfer - ISM: cosmic rays

\section{Introduction}

The most rigorous treatment of cosmic ray $(\mathrm{CR})$ propagation in the interplanetary medium and in the Galaxy is based on the kinetic equation (Earl 1976; Dorman \& Katz 1977; Achatz et al. 1991; Fedorov et al. 1992; Webb et al. 2000). The kinetic equation describes the transport of energetic particles in the magnetic field, which can be presented as a superposition of a mean regular field and magnetic irregularities of various scales. The regular interplanetary magnetic field has a spiral configuration and decreases with heliocentric distance. This spatial variation of a regular magnetic field causes CR focusing (Earl 1976; Bazilevskaja \& Golynskaja 1989), and magnetic fluctuations produce the scattering of charged energetic particles (Dorman \& Katz 1977; Toptygin 1985; Achatz et al. 1991; Schlickeiser et al. 1991).

In the present paper we assume a mean magnetic field to be homogeneous and sufficiently strong, thus the drift approximation is applicable to the particle motion description. We do not take into consideration the process of particle cross field diffusion (Dorman et al. 1990; Chuvilgin \& Ptuskin 1993; Kirk et al. 1996; Michalek \& Ostrowski 1997) and convection effects in CR, which are due to the interaction of energetic particles with moving solar wind plasma (Earl 1984; Toptygin 1985; Achatz et al. 1991; Fedorov et al. 1992).

CR scattering on magnetic field irregularities can be treated as particle interaction with "magnetic clouds" and can be described by the Boltzmann collision integral (Gleeson \& Axford 1967; Dolginov \& Toptygin 1967; Fisk \& Axford 1969;

Send offprint requests to: Yu. I. Fedorov, e-mail: fedorov@mao.kiev.ua
Toptygin 1985). The Boltzmann kinetic equation can be solved analytically and the exact expression for a Green function can be obtained, which describes CR distribution after an impulsive injection of particles with a given velocity direction (Fedorov \& Shakhov 1993, 1994). Kota (1994) has derived an exact formula for particle density under isotropic, instantaneous particle release and has also considered CR transport in the case of anisotropic scattering. Recently, the multiple scattering approach for the kinetic equation solution was developed (Webb et al. 2000), which can be succesfully applied to a number of problems in physical kinetics.

The diffusion approximation, widely used in the theory of CR transport, proved to be deficient in a number of problems (Earl 1973; Toptygin 1985). The telegraph equation description was applied to the process of solar CR propagation by many authors (Shishov 1966; Fisk \& Axford 1969; Earl 1973; Gombosi et al. 1993). Recently, the approximate methods of the kinetic equation solution, based on the investigation of a set of equations for harmonics of the CR distribution function, were developed (Earl 1994; Zank et al. 1999). The accuracy of these methods was explored by comparison with exact analytical solutions of the Boltzmann equation and with numerical results of Monte Carlo simulations (Earl 1994; Zank et al. 1999).

In the present paper we consider the propagation of energetic particles under their instantaneous, isotropic injection into scattering medium with a homogeneous regular magnetic field. Starting from the Boltzmann equation, the exact analytical expressions for particle density and anisotropy are derived and approximate formulas valid for late times with respect to the scattering timescale are obtained (Sect. 2). In Sect. 3 the set of equations for harmonics of CR distribution function is 
derived and telegraph equations for particle density and flux are obtained and solved. The derivation of a new transport equation taking into account the second harmonic of the distribution function is carried out and the solution of this equation is given in Sect. 4. The approximate expressions valid for late times are considered and results of calculations are compared to the exact analytical solutions of the Boltzmann equation. The CR transport governed by the Fokker-Planck equation is studied in Sect. 5. The derivation of a new transport equation describing multiple small angle scattering is carried out. In Sect. 6 the modified telegraph equations, corresponding to Boltzmann and Fokker-Planck equations, are obtained and solutions of these equations are analyzed.

In Sect. 7 the transport equation, taking into consideration anisotropic particle scattering on magnetic irregularities and solar cosmic ray (SCR) focusing in the interplanetary magnetic field, is derived. The solutions of the obtained equations are applied to the analysis of the solar proton event of July 14, 2000.

\section{Kinetic equation solution}

Let us start from the kinetic equation describing charged particle propagation in the interplanetary magnetic field (Toptygin 1985; Fedorov \& Shakhov 1994; Kota 1994)

$\frac{\partial f}{\partial t}+v \mu \frac{\partial f}{\partial z}+v_{\mathrm{s}} f-\frac{v_{\mathrm{s}}}{2} \int_{-1}^{1} \mathrm{~d} \mu f=\frac{1}{2} \delta(z) \delta(t)$,

where $f(z, t, \mu)$ is CR distribution function, $v_{\mathrm{s}}$ is a collision frequency, describing interaction of particles with magnetic irregularities ("magnetic clouds"), and $\mu$ is a particle pitch angle cosine. The instantaneous isotropic source of particles is included in the right hand side of Eq. (1). It is supposed that the CR distribution function depends only on coordinate $z$ along a uniform regular magnetic field.

After introduction of dimensionless variables

$y=\frac{z}{\Lambda} ; \tau=\frac{v t}{\Lambda}$

(where $\Lambda=v / v_{\mathrm{s}}$ is the particle mean free path) the kinetic Eq. (1) can be rewritten in the form

$\frac{\partial f}{\partial \tau}+\mu \frac{\partial f}{\partial y}+f-\frac{1}{2} \int_{-1}^{1} \mathrm{~d} \mu f=\frac{1}{2 \Lambda} \delta(y) \delta(\tau)$.

It is known that the exact solution of kinetic Eq. (2) can be separated into those particles which have not experienced scattering, and scattered ones (Fedorov \& Shakhov 1993; Kota 1994; Fedorov et al. 1995; Webb et al. 2000). The unscattered distribution function, associated with kinetic Eq. (2), has the form

$f^{0}(y, \tau, \mu)=\frac{\exp (-\tau)}{2 \Lambda} \delta(y-\mu \tau)$

According to this equation the unscattered particles with pitch angle $\vartheta$ in time $\tau$ are located in a position $y=\mu \tau$.

The solution of kinetic Eq. (2) can be reached by using a Laplace transform in dimensionless time $\tau$ and a Fourier transform in dimensionless spatial coordinate $y$ (Fedorov \& Shakhov 1993; Fedorov et al. 1995). The CR distribution function can be presented as an expansion into Legendre polynomials

$f(y, \tau, \mu)=\sum_{n=0} \frac{2 n+1}{2} f_{n}(y, \tau) P_{n}(\mu)$,

where quantities

$f_{n}(y, \tau)=\int_{-1}^{1} \mathrm{~d} \mu P_{n}(\mu) f(y, \tau, \mu)$

represent harmonics of the particle distribution function. In the present paper we shall deal only with particle density $f_{0}$ and particle flux $f_{1}$ as functions of time and position.

The density of unscattered particles can be obtained by integration of distribution function (3) over $\mu$

$f_{0}^{0}(y, \tau)=\frac{\exp (-\tau)}{2 \Lambda \tau}\{\Theta(y+\tau)-\Theta(y-\tau)\}$,

where $\Theta(x)$ is the Heaviside step function. According to (6) the unscattered particles are extended in the region $-\tau \leq y \leq \tau$ and the number of these particles decays exponentially with time after injection. Multiplying formula (3) by $\mu$ and integrating over $\mu$ we obtain the following expression for the first harmonic of the unscattered distribution function

$f_{1}^{0}(y, \tau)=\frac{y \exp (-\tau)}{2 \Lambda \tau^{2}}\{\Theta(y+\tau)-\Theta(y-\tau)\}$.

The scattered particle density can be written as (Kota 1994)

$$
\begin{aligned}
f_{0}^{s}(y, \tau)= & \frac{1}{\pi \Lambda} \int_{0}^{\pi / 2} \mathrm{~d} k \frac{k^{2} \cos k y \exp (\tau k \cot k-\tau)}{\sin ^{2} k} \\
& +\frac{\exp (-\tau)}{4 \pi \Lambda} \int_{-1}^{1} \mathrm{~d} \eta \exp \left(\frac{\zeta \kappa}{2}\right)\left\{2 \pi \kappa \cos \frac{\pi \zeta}{2}\right. \\
& \left.+\left(\kappa^{2}-\pi^{2}\right) \sin \frac{\pi \zeta}{2}\right\}\{\Theta(\zeta) \Theta(\eta)-\Theta(-\zeta) \Theta(-\eta)\}
\end{aligned}
$$

where $\kappa=\ln \frac{1-\eta}{1+\eta} ; \zeta=y-\tau \eta$. Note that the derivation of this formula is presented in Appendix A.

The spatial distribution of particles for $\tau=3$ is shown in Fig. 1, where the dashed curve corresponds to unscattered particles (6), the dotted curve accounts for scattered particles (8), and the curve 1 gives the density of all particles. One can see that the density of unscattered particles is uniform in the region $y<\tau$ and the scattered particle density decreases with $y$. It is worth noting that the density equals zero for $y>\tau$ in accordance with the finite value of particle velocity.

The temporal profile of particle density at position $y=2$ is demonstrated in Fig. 2. One can see that the number of unscattered particles (presented by dashed curve ) at $\tau=y$ increases drastically from zero to a finite value, and at $\tau>y$ the unscattered particle density decays exponentially. The density of scattered particles at $\tau>y$ increases continuously from zero at $\tau=y$, and after a couple of scattering times the scattered particles dominate the distribution of all particles, presented in Fig. 2 by curve 1 .

Let us consider the particle distribution at late times, when the time after injection is much longer than the mean time between collisions $(\tau \gg 1)$. In this case one can neglect the unscattered particle density (6) and the second term in expression (8), which is small because of the exponential multiplier. 


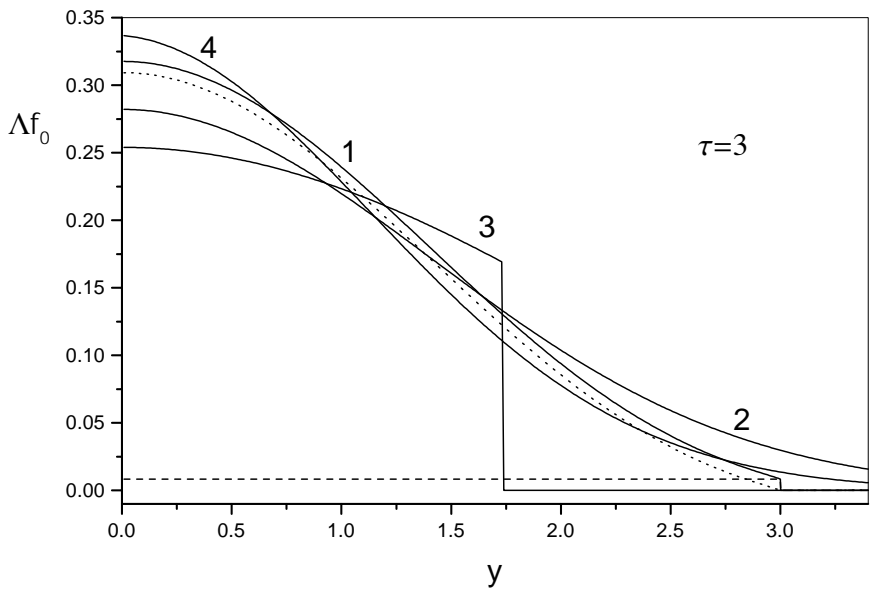

Fig. 1. The dependence of density on the spatial coordinate $y(\tau=3)$, curve 1 - kinetic equation solution, curve 2 - diffusion approximation, curve 3 - telegraph equation solution, curve 4 - solution of transport Eq. (27), dashed curve - unscattered particles, dotted curve - scattered particles.

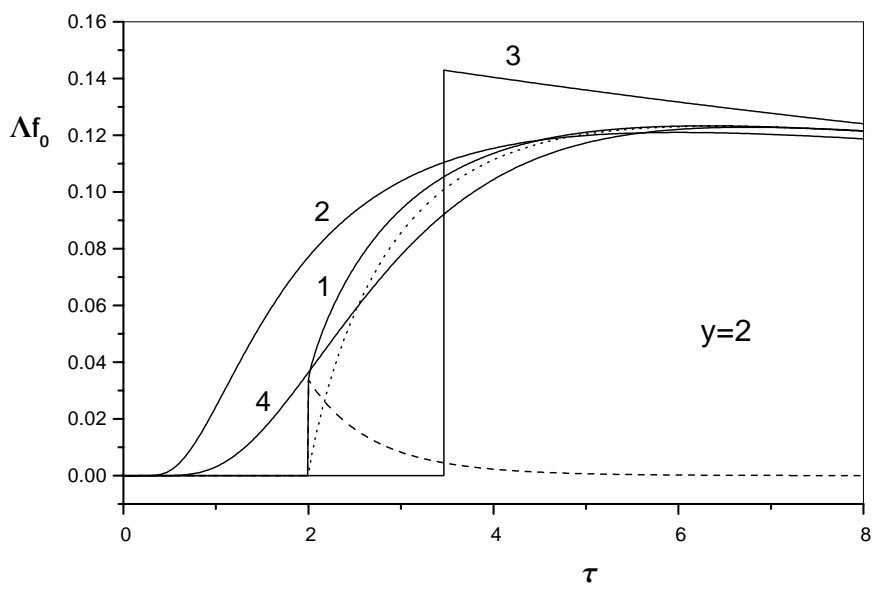

Fig. 2. The temporal dependence of density in $y=2$ position, curve $1-$ kinetic equation solution, curve 2 - diffusion approximation, curve 3 telegraph equation solution, curve 4 - solution of transport Eq. (27), dashed curve - unscattered particles, dotted curve - scattered particles.

Thus under condition $\tau \gg 1$ we can obtain from Eq. (8) the following approximate formula (the derivation of this expression is done in Appendix A)

$f_{0}(y, \tau)=\frac{\sqrt{3} \exp \left(-\frac{3 y^{2}}{4 \tau}\right)}{2 \Lambda \sqrt{\pi \tau}}\left\{1+\frac{7}{20 \tau}-\frac{3 y^{2}}{10 \tau^{2}}\right\}$.

Note that the well known solution of the diffusion equation can be obtained from (9) by retaining only the first term in brackets. The diffusion density is shown in Figs. 1, 2 by curve 2; one can see from these figures that the kinetic equation solution (curve 1) approaches the diffusion density with increasing time after particle injection.

The derivation of an expression for the first harmonic of the distribution function from kinetic Eq. (2) can be done in a similar manner and is presented in Appendix A. The first harmonic

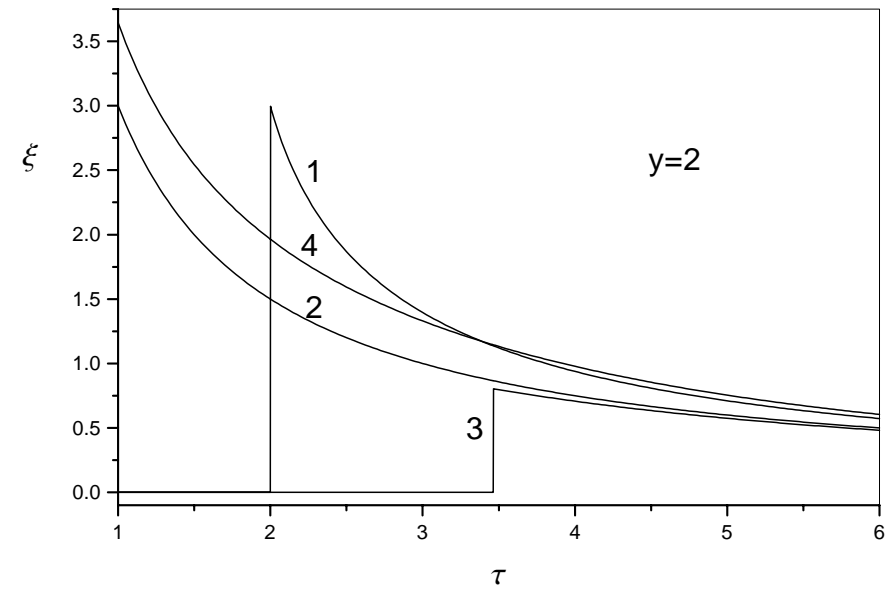

Fig. 3. The temporal dependence of anisotropy $\xi$ in $y=2$ position, curve 1 - kinetic equation solution, curve 2 - diffusion approximation, curve 3 - telegraph equation solution, curve 4 - solution of new transport equation.

of the scattered distribution function has the form

$$
\begin{aligned}
f_{1}^{s}(y, \tau)= & \frac{1}{\pi \Lambda} \int_{0}^{\pi / 2} \mathrm{~d} k \\
& \times \frac{(\sin k-k \cos k) k \sin k y \exp (\tau k \cot k-\tau)}{\sin ^{3} k} \\
& +\frac{\exp (-\tau)}{4 \pi \Lambda} \int_{-1}^{1} \mathrm{~d} \eta \exp \left(\frac{\zeta \kappa}{2}\right) \\
& \times\left\{\left(2 \kappa+\eta \kappa^{2}-\eta \pi^{2}\right) \sin \frac{\pi \zeta}{2}+2 \pi(1+\eta \kappa) \cos \frac{\pi \zeta}{2}\right\} \\
& \times\{\Theta(\zeta) \Theta(\eta)-\Theta(-\zeta) \Theta(-\eta)\} .
\end{aligned}
$$

The anisotropy of CR distribution is defined by the formula

$\xi(y, \tau)=\frac{3 f_{1}(y, \tau)}{f_{0}(y, \tau)}$

and can be calculated starting from equations for $f_{1}(7),(10)$ and from Eqs. (6), (8) for particle density. At late times after particle release the expression (10) can be simplified and the approximate formula for the first harmonic can be obtained (Appendix A). Starting with expressions (A.13) and (9) we can write the formula for anisotropy valid under condition $\tau \gg 1$

$\xi(y, \tau)=\frac{3 y}{2 \tau}\left\{1+\frac{7}{10 \tau}+\frac{3 y^{2}}{20 \tau^{2}}\right\}$.

The anisotropy value in the diffusion approximation can be obtained from Eq. (12) by neglecting the two last terms in the brackets. Figure 3 illustrates the time evolution of particle anisotropy (11) for $y=2$; in this figure curve 1 corresponds to the anisotropy value calculated according expressions (6)(8), (10), derived from kinetic equation, and curve 2 shows $\xi$ in the diffusion approximation. It is instructive to note that the first arriving particles $(\tau=y)$ offer the maximal anisotropy value $\xi=3$. One can see from Figs. $1-3$ that the diffusion approximation is applicable only for late times after injection, and the anisotropy calculated on the basis of the kinetic equation at all times exceeds the corresponding diffusion value. 


\section{Telegraph equation description}

The set of equations for harmonics of CR distribution function can be obtained by multiplying the kinetic equation by $P_{n}(\mu)$ and by integrating the result from $\mu=-1$ to $\mu=1$ (Earl 1994; Zank et al. 1999). If we multiply Eq. (2) by $1, \mu$ and $P_{2}(\mu)$ respectively and integrate over $\mu$ we obtain the following set of equations

$\frac{\partial f_{0}}{\partial \tau}+\frac{\partial f_{1}}{\partial y}=\frac{1}{\Lambda} \delta(y) \delta(\tau)$

$\frac{\partial f_{1}}{\partial \tau}+f_{1}+\frac{1}{3} \frac{\partial f_{0}}{\partial y}+\frac{2}{3} \frac{\partial f_{2}}{\partial y}=0$

$\frac{\partial f_{2}}{\partial \tau}+f_{2}+\frac{2}{5} \frac{\partial f_{1}}{\partial y}+\frac{3}{5} \frac{\partial f_{3}}{\partial y}=0$

We can obtain the diffusion approximation if we neglect the first term in (14) assuming that the time evolution of particle distribution occurs sufficiently slowly so that $\frac{\partial f_{1}}{\partial \tau} \ll f_{1}$, although neglecting the second harmonic in comparison to $f_{0}$. In this case the particle flux is proportional to the density gradient

$f_{1}(y, \tau)=-\frac{1}{3} \frac{\partial f_{0}(y, \tau)}{\partial y}$,

and Eq. (13) takes the form

$$
\frac{\partial f_{0}}{\partial \tau}-\frac{1}{3} \frac{\partial^{2} f_{0}}{\partial y^{2}}=\frac{1}{\Lambda} \delta(y) \delta(\tau)
$$

The solution of the diffusion equation is well known

$f_{0}(y, \tau)=\frac{\sqrt{3} \exp \left(-\frac{3 y^{2}}{4 \tau}\right)}{2 \Lambda \sqrt{\pi \tau}}$,

and is presented by curve 2 in Figs. 1, 2. The particle anisotropy $\xi(11)$ in the diffusion approximation is given by formula

$\xi(y, \tau)=\frac{3 y}{2 \tau}$.

Curve 2 in Fig. 3 illustrates the evolution of the diffusion anisotropy (19), which decays inversely proportional to time.

If we neglect the second harmonic in Eq. (14) but retain the time derivative of $f_{1}$ we obtain the well-known telegraph equation (Shishov 1966; Fisk \& Axford 1969; Earl 1973; Dorman et al. 1983; Gombosi et al. 1993)

$$
\frac{\partial^{2} f_{0}}{\partial \tau^{2}}+\frac{\partial f_{0}}{\partial \tau}-\frac{1}{3} \frac{\partial^{2} f_{0}}{\partial y^{2}}=\frac{1}{\Lambda}\left\{\delta(y) \delta(\tau)+\delta(y) \delta^{\prime}(\tau)\right\}
$$

The solution of telegraph Eq. (20) is presented in Appendix B and has the following form

$$
\begin{aligned}
f_{0}(y, \tau)= & \frac{\sqrt{3} \exp \left(-\frac{\tau}{2}\right)}{4 \Lambda}\left\{\left[I_{0}\left(\frac{\xi}{2}\right)+\frac{\tau}{\xi} I_{1}\left(\frac{\xi}{2}\right)\right]\right. \\
& \times \Theta(\tau-\sqrt{3}|y|)+2 \delta(\tau-\sqrt{3}|y|)\},
\end{aligned}
$$

where $\xi=\sqrt{\tau^{2}-3 y^{2}}$, and $I_{n}(x)$ stands for the modified Bessel function of the $n$th order.
The solution of the telegraph Eq. (21) is illustrated in Figs. 1, 2 by curve 3 . It is worth mentioning that in these figures we do not present the singular part of the solution (21), which is proportional to $\delta$-function. One can see from formula (21) and from Fig. 1 (curve 3 ) that in this approximation at time $\tau$ the particles are located only in region $-v t / \sqrt{3}<y<v t / \sqrt{3}$ and this region expands with time with characteristic velocity $v / \sqrt{3}$. It is instructive to note that the telegraph equation approximation corresponds to the speed of disturbances propagation $v / \sqrt{3}$ (Shishov 1966; Fisk et al. 1969; Gombosi et al. 1993). This value of signal speed as well as the pulses, propagating on the forward fronts of particle distribution, result from higher harmonics neglected in the derivation of the telegraph Eq. (20). One can see from Fig. 2, where the particle density (21) is displayed as a function of time $\tau$, that the particles appear in $y$-position impulsively at $\tau=\sqrt{3} y$. It is instructive to note that the description based on the commonly used telegraph Eq. (20) does not improve the accuracy of density and anisotropy calculations in comparison with the diffusion approximation, in contrast to the modified telegraph equation derived by Gombosi et al. (1993).

At late times, after particles have undergone many scatters, one can obtain from (21) the following approximate formula (see Appendix B)

$f_{0}(y, \tau)=\frac{\sqrt{3} \exp \left(-\frac{3 y^{2}}{4 \tau}\right)}{2 \Lambda \sqrt{\pi \tau}}\left\{1-\frac{1}{4 \tau}+\frac{3 y^{2}}{2 \tau^{2}}\right\}$.

Note that expression (22) differs from formula (9), based on the kinetic equation.

Neglecting the second harmonic of distribution function in the set of Eqs. (13), (14) one can obtain the telegraph equation for particle flux

$$
\frac{\partial^{2} f_{1}}{\partial \tau^{2}}+\frac{\partial f_{1}}{\partial \tau}-\frac{1}{3} \frac{\partial^{2} f_{1}}{\partial y^{2}}=-\frac{1}{3 \Lambda} \delta^{\prime}(y) \delta(\tau) .
$$

The solution of this equation, which can be derived quite similarly to the solution of the telegraph Eq. (20), has the form

$$
\begin{array}{r}
f_{1}(y, \tau)=\frac{\exp \left(-\frac{\tau}{2}\right)}{2 \Lambda}\left\{\frac{\sqrt{3} y}{2 \xi} I_{1}\left(\frac{\xi}{2}\right) \Theta(\tau-\sqrt{3}|y|)\right. \\
\left.+\frac{y}{|y|} \delta(\tau-\sqrt{3}|y|)\right\}
\end{array}
$$

The anisotropy of particle distribution $\xi(11)$, calculated from (21), (24), is shown in Fig. 3 (curve 3). Note that this value of anisotropy is fairly close to the value of $\xi$ in the diffusion approximation (19) if $\tau>\sqrt{3} y$. At late times $(\tau \gg 1)$ one can derive from $(22),(24)$ the following formula

$\xi(y, \tau)=\frac{3 y}{2 \tau}\left\{1-\frac{1}{2 \tau}+\frac{3 y^{2}}{4 \tau^{2}}\right\}$

which is different from expression (12), obtained from the kinetic equation. 


\section{New transport equation}

In order to derive the CR transport equation, including the second harmonic of distribution function, we neglect the first and the last terms in Eq. (15). Thus we suppose that the distribution function evolves quite slowly with time, so $\frac{\partial f_{2}}{\partial \tau} \ll f_{2}$, and that the third harmonic is small in comparison with the first one. In this approximation the second harmonic is proportional to the gradient of $f_{1}$ :

$f_{2}(y, \tau)=-\frac{2}{5} \frac{\partial f_{1}(y, \tau)}{\partial y}$

Substituting (26) in (14) with using Eq. (13) yields the following transport equation

$$
\begin{aligned}
\frac{\partial^{2} f_{0}}{\partial \tau^{2}}+\frac{\partial f_{0}}{\partial \tau}- & \frac{1}{3} \frac{\partial^{2} f_{0}}{\partial y^{2}}-\frac{4}{15} \frac{\partial^{3} f_{0}}{\partial y^{2} \partial \tau} \\
& =\frac{1}{\Lambda}\left\{\delta(y) \delta(\tau)+\delta(y) \delta^{\prime}(\tau)-\frac{4}{15} \delta^{\prime \prime}(y) \delta(\tau)\right\}
\end{aligned}
$$

The solution of (27) can be obtained by performing Laplace and Fourier transform methods (Appendix C), and this solution can be written in the form

$$
\begin{aligned}
f_{0}(y, \tau)= & \frac{\exp \left(-\frac{\tau}{2}\right)}{\pi \Lambda} \int_{0}^{\infty} \mathrm{d} k \cos k y \exp \left(-\frac{2}{15} k^{2} \tau\right) \\
& \times\left\{\left(k^{2}+\frac{15}{4}\right) \frac{\sinh \left(\frac{2}{15} \tau \sqrt{\Psi}\right)}{\sqrt{\Psi}}+\cosh \left(\frac{2}{15} \tau \sqrt{\Psi}\right)\right\},
\end{aligned}
$$

with

$\Psi=k^{4}-\frac{45}{4} k^{2}+\frac{225}{16}$.

The relationship (28) is illustrated in Figs. 1, 2 by curve 4. One can see from Fig. 2 that obtained expression (28) approximates the kinetic equation solution (7), (8) (curve 1) fairly well under condition $\tau>y$, but the density (28) differs from zero in the region $y>\tau$. The nonzero value of $f_{0}$ in this region is caused by using approximate formula (26) instead of Eq. (15).

Let us consider particle distribution (28) long after injection. Under condition $\tau \gg 1$ the integrand in (28) has a sharp maximum at $k=0$, so it can be expanded into series near $k=0$ (see Appendix A). Then integrating the obtained expression (A.11) over $k$ we obtain formula (9). It is interesting to note that at late times the same relationship (9) follows both from the solution of new transport Eq. (28) and from the kinetic equation solution (8). Thus a new transport Eq. (27) describes particle propagation with good accuracy if the time after injection exceeds both a mean scattering time and the time of unscattered particle propagation from the source $(t>\Lambda / v ; t>z / v)$.

Starting from the set of Eqs. (13), (14), (26) one can obtain the following equation for particle flux

$\frac{\partial^{2} f_{1}}{\partial \tau^{2}}+\frac{\partial f_{1}}{\partial \tau}-\frac{1}{3} \frac{\partial^{2} f_{1}}{\partial y^{2}}-\frac{4}{15} \frac{\partial^{3} f_{1}}{\partial y^{2} \partial \tau}=-\frac{1}{3 \Lambda} \delta^{\prime}(y) \delta(\tau)$.

Note that the only difference between equation for $f_{0}(27)$ and transport Eq. (30) consists of a source term. So, we can obtain the solution of (30) in the same manner as expression (28) (see Appendix C), and this solution has the form

$$
\begin{aligned}
f_{1}(y, \tau)= & \frac{5 \exp \left(-\frac{\tau}{2}\right)}{2 \pi \Lambda} \int_{0}^{\infty} \mathrm{d} k k \sin k y \\
& \times \exp \left(-\frac{2}{15} k^{2} \tau\right) \frac{\sinh \left(\frac{2}{15} \tau \sqrt{\Psi}\right)}{\sqrt{\Psi}} .
\end{aligned}
$$

The curve 4 in Fig. 3 shows particle anisotropy (11), calculated on the basis of (28), (31), as a function of time in the case $y=2$. The anisotropy of the first arriving particles in this approximation appears to be smaller than the corresponding value $\xi=3$, obtained from the kinetic equation (curve 1). One can see from Fig. 3 that the accuracy of the anisotropy calculation given by relationships (28), (31) (curve 4) is higher than the accuracy of $\xi$ in the diffusion approximation or in the telegraph equation description (curve 2 and curve 3, correspondingly). At late times after particle release expression (31) simplifies to (A.12), from which one can obtain formula (12), derived from the kinetic equation solution.

\section{Multiple small angle scattering}

Now we consider the kinetic equation, describing multiple scattering of particles on magnetic irregularities (Earl 1976; Toptygin 1985; Achatz et al. 1991)

$\frac{\partial f}{\partial t}+v \mu \frac{\partial f}{\partial z}-\frac{\partial}{\partial \mu} D_{\mu \mu} \frac{\partial f}{\partial \mu}=\frac{1}{2} \delta(z) \delta(t)$,

where $D_{\mu \mu}$ represents the diffusion coefficient in angular space. Under isotropic scattering, the diffusion coefficient is given by (Earl 1976; Schlickeiser et al. 1991)

$D_{\mu \mu}=\frac{v}{2 \Lambda}\left(1-\mu^{2}\right)$,

where $\Lambda$ is the particle mean free path.

Using the dimensionless variables the Fokker-Planck Eq. (32) can be rewritten as

$\frac{\partial f}{\partial \tau}+\mu \frac{\partial f}{\partial y}-\frac{1}{2} \frac{\partial}{\partial \mu}\left(1-\mu^{2}\right) \frac{\partial f}{\partial \mu}=\frac{1}{2 \Lambda} \delta(y) \delta(\tau)$.

The set of equations for harmonics of distribution function can be obtained by multiplying Eq. (34) by $P_{n}(\mu)$ and by integrating the result over $\mu$. The first two equations are given by formulas (13), (14), and the third equation of the set has the form

$\frac{\partial f_{2}}{\partial \tau}+3 f_{2}+\frac{2}{5} \frac{\partial f_{1}}{\partial y}+\frac{3}{5} \frac{\partial f_{3}}{\partial y}=0$

This equation differs from (15) only by the coefficient at the second harmonic. Equations (13) and (14) can be obtained both from Boltzmann equation and from Fokker-Planck equation. Thus, if we neglect the second harmonic in (14) we can obtain the same diffusion Eq. (17) or telegraph Eq. (20) from kinetic Eqs. (2) and (34).

In order to derive the transport equation, including the second spherical harmonic, we suppose that the second harmonic 


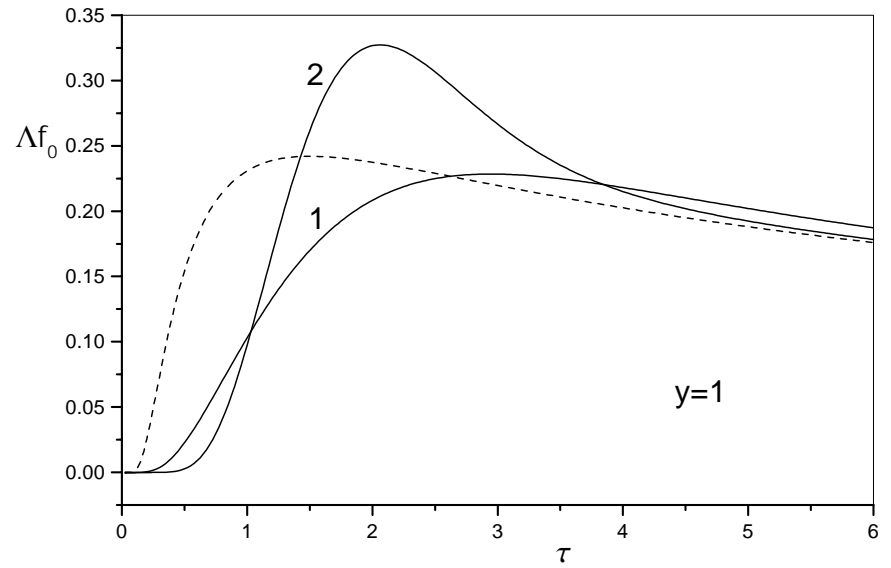

Fig. 4. The temporal dependence of particle density in $y=1$ position, curve 1 - solution of transport Eq. (27), curve 2 - solution of transport Eq. (37), dashed curve - diffusion approximation.

varies sufficiently slowly with time and we neglect the third harmonic in (35). The Eq. (35) can then be written as

$f_{2}(y, \tau)=-\frac{2}{15} \frac{\partial f_{1}(y, \tau)}{\partial y}$

So in this approximation the second harmonic is proportional to the gradient of $f_{1}$. It is worth mentioning that a multiplier in the right hand side of (36) is 3 times less than in the analogous expression (26). From the set of Eqs. (13), (14), (36) one can obtain the following transport equation

$$
\begin{aligned}
\frac{\partial^{2} f_{0}}{\partial \tau^{2}}+ & \frac{\partial f_{0}}{\partial \tau}-\frac{1}{3} \frac{\partial^{2} f_{0}}{\partial y^{2}}-\frac{4}{45} \frac{\partial^{3} f_{0}}{\partial y^{2} \partial \tau} \\
& =\frac{1}{\Lambda}\left\{\delta(y) \delta(\tau)+\delta(y) \delta^{\prime}(\tau)-\frac{4}{45} \delta^{\prime \prime}(y) \delta(\tau)\right\}
\end{aligned}
$$

This equation differs from the analogous Eq. (27) only by the coefficient at the mixed derivative. Thus, the solution of (37) can be obtained quite similar to the solution of (27) (which is derived in Appendix C), and can be written as

$$
\begin{aligned}
f_{0}(y, \tau)= & \frac{\exp \left(-\frac{\tau}{2}\right)}{\pi \Lambda} \int_{0}^{\infty} \mathrm{d} k \cos k y \exp \left(-\frac{2}{45} k^{2} \tau\right) \\
& \times\left\{\left(k^{2}+\frac{45}{4}\right) \frac{\sinh \left(\frac{2}{45} \tau \sqrt{\Psi_{1}}\right)}{\sqrt{\Psi_{1}}}+\cosh \left(\frac{2}{45} \tau \sqrt{\Psi_{1}}\right)\right\},
\end{aligned}
$$

with

$\Psi_{1}=k^{4}-\frac{585}{4} k^{2}+\frac{2025}{16}$.

As in relationship (28), the integration interval in (38) should be divided into three parts. If $k<k_{1}$ or $k>k_{2}$ the integrand is defined as in formula (38), but in the case $k_{1}<k<k_{2}$ it is necessary to replace in (38) the functions sinh, cosh by $\sin$, cos, and to change the sign of $\Psi_{1}$ (39) (see Appendix C). The quantities $k_{1}, k_{2}$ are defined by relations:

$k_{1}^{2}=\frac{45}{8}(13-\sqrt{165}) ; k_{2}^{2}=\frac{45}{8}(13+\sqrt{165})$.
The expression (38) can be rewritten in the form

$$
\begin{aligned}
f_{0}(y, \tau)= & \frac{\exp \left(-\frac{\tau}{2}\right)}{\pi \Lambda} \int_{0}^{\infty} \mathrm{d} k \cos k y\left\{\exp \left(-\frac{2}{45} k^{2} \tau\right)\right. \\
& \times\left[\left(k^{2}+\frac{45}{4}\right) \frac{\sinh \left(\frac{2}{45} \tau \sqrt{\Psi_{1}}\right)}{\sqrt{\Psi_{1}}}+\cosh \left(\frac{2}{45} \tau \sqrt{\Psi_{1}}\right)\right] \\
& \left.-\exp \left(-\frac{13}{4} \tau\right)\right\}+\frac{\exp \left(-\frac{15}{4} \tau\right)}{\Lambda} \delta(y)
\end{aligned}
$$

Note that from Eq. (41) the occurrence of a singularity in $y=0$ is evident.

One can show that at late times the expression for particle density (41) simplifies to

$$
f_{0}(y, \tau)=\frac{\sqrt{3} \exp \left(-\frac{3 y^{2}}{4 \tau}\right)}{2 \Lambda \sqrt{\pi \tau}}\left\{1-\frac{1}{20 \tau}+\frac{9 y^{2}}{10 \tau^{2}}\right\} .
$$

Formula (42) is different from expression (9), resulting from kinetic Eq. (2), which corresponds to CR scattering on "magnetic clouds" (hard sphere scattering). In Fig. 4 particle density versus dimensionless time in $y=1$ position is shown. The dashed curve corresponds to the diffusion approximation (18), the curve 1 illustrates solution (28) of the transport Eq. (27), and the curve 2 presents formula (38), describing the solution of Eq. (37). It is interesting to note that multiple small angle scattering is characterized by sharper maximum and earlier phase of maximal intensity in comparison with the case of hard sphere scattering (Fig. 4).

Starting from the set of Eqs. (13), (14), (36) one can obtain the following equation for the first harmonic of the distribution function

$\frac{\partial^{2} f_{1}}{\partial \tau^{2}}+\frac{\partial f_{1}}{\partial \tau}-\frac{1}{3} \frac{\partial^{2} f_{1}}{\partial y^{2}}-\frac{4}{45} \frac{\partial^{3} f_{1}}{\partial y^{2} \partial \tau}=-\frac{1}{3 \Lambda} \delta^{\prime}(y) \delta(\tau)$.

Equation (43) differs from the analogous Eq. (30), derived from kinetic Eq. (2), only by the coefficient at the mixed derivative, and the solution of (43) can be written in the form

$$
\begin{aligned}
f_{1}(y, \tau)= & \frac{15 \exp \left(-\frac{\tau}{2}\right)}{2 \pi \Lambda} \int_{0}^{\infty} \mathrm{d} k k \sin k y \\
& \times \exp \left(-\frac{2}{45} k^{2} \tau\right) \frac{\sinh \left(\frac{2}{45} \tau \sqrt{\Psi_{1}}\right)}{\sqrt{\Psi_{1}}} .
\end{aligned}
$$

We can rewrite expression (44) as following

$$
\begin{aligned}
f_{1}(y, \tau)= & \frac{15 \exp \left(-\frac{\tau}{2}\right)}{2 \pi \Lambda} \int_{0}^{\infty} \mathrm{d} k k \sin k y \\
& \times\left\{\exp \left(-\frac{2}{45} k^{2} \tau\right) \frac{\sinh \left(\frac{2}{45} \tau \sqrt{\Psi_{1}}\right)}{\sqrt{\Psi_{1}}}-\frac{\exp \left(-\frac{13}{4} \tau\right)}{2 k^{2}}\right\} \\
& +\frac{15}{4 \Lambda} \exp \left(-\frac{15}{4} \tau\right)\left\{\Theta(y)-\frac{1}{2}\right\}
\end{aligned}
$$

It is obvious from this formula that the impulsive variation of $f_{1}$ occurs at $y=0$ (see also relationship (C.7)).

The calculations show that the anisotropy (11), obtained by using formulas (38), (44), is somewhat higher than the 
corresponding value calculated in accordance with expressions (28), (31). Thus under the same value of particle mean free path the higher anisotropy value and more pronounced maximum of temporal intensity profile is typical in the case of small angle scattering in comparison with hard sphere scattering (Fig. 4). It is instructive to note that in this approximation the anisotropy of first arriving particles $(\tau=y)$ takes the value somewhat below the maximal level of $\xi=3$, obtained from the kinetic equation.

At late times after particle injection from Eqs. (42), (44) we obtain the approximate formula

$\xi(y, \tau)=\frac{3 y}{2 \tau}\left\{1-\frac{1}{10 \tau}+\frac{11 y^{2}}{20 \tau^{2}}\right\}$,

which is different from expression (12) obtained from kinetic Eq. (2).

\section{Modified telegraph equation}

The new transport equations derived in this paper are associated with modified telegraph equations obtained by Gombosi et al. (1993). It is interesting to emphasize that the same diffusion Eq. (17) and telegraph Eq. (20) follow from the Boltzmann Eq. (2) as well as from the Fokker-Planck Eq. (34) if the corresponding mean free path values coincide. However, the transport Eqs. (27) and (37) associated with kinetic Eqs. (2) and (34) are different from one another. It was demonstrated by Gombosi et al. (1993) that more rigorous derivation leads to the form of the telegraph equation that is different from (20).Thus in the case of large angle scattering the signal speed in modified telegraph equation equals $v \sqrt{\frac{5}{3}}$ in contrast to the $\frac{v}{\sqrt{3}}$ value of disturbances propagation speed in Eq. (20). Under small angle scattering one can obtain the modified telegraph equation with the characteristic propagation speed of $v \sqrt{\frac{5}{11}}$, which is below the particle velocity value (Gombosi et al. 1993). Let us transform the transport Eq. (27) in the telegraph equation form. In order to replace a mixed derivative in (27) one can use the diffusion equation. Differentiating (17) we can obtain the approximate relation for the mixed derivative which appears to be proportional to the second order time derivative of particle density. Substituting the obtained expression into transport Eq. (27) yields

$$
\begin{aligned}
\frac{1}{5} \frac{\partial^{2} f_{0}}{\partial \tau^{2}}+ & \frac{\partial f_{0}}{\partial \tau}-\frac{1}{3} \frac{\partial^{2} f_{0}}{\partial y^{2}} \\
& =\frac{1}{\Lambda}\left\{\delta(y) \delta(\tau)+\frac{1}{5} \delta(y) \delta^{\prime}(\tau)-\frac{4}{15} \delta^{\prime \prime}(y) \delta(\tau)\right\}
\end{aligned}
$$

Hence starting from transport Eq. (27) we reach the modified telegraph Eq. (47), derived in the paper of Gombosi et al. (1993).
The solution of the modified telegraph Eq. (47), which can be obtained quite similarly to the solution of (20) (Appendix B), is given by

$$
\begin{aligned}
f_{0}(y, \tau)= & \frac{\sqrt{15} \exp \left(-\frac{5}{2} \tau\right)}{4 \Lambda}\left\{\left[I_{0}\left(\frac{5}{2} \xi_{1}\right)\right.\right. \\
& +\frac{\tau}{\xi_{1}}\left(1+\frac{4 \tau}{5 \xi_{1}^{2}}\right) I_{1}\left(\frac{5}{2} \xi_{1}\right)-\frac{3 y^{2}}{5 \xi_{1}^{2}}\left[I_{0}\left(\frac{5}{2} \xi_{1}\right)\right. \\
& \left.\left.+I_{2}\left(\frac{5}{2} \xi_{1}\right)\right]\right] \Theta\left(\tau-\sqrt{\frac{3}{5}}|y|\right) \\
& \left.+2\left(\frac{1}{5}-\tau\right) \delta\left(\tau-\sqrt{\frac{3}{5}}|y|\right)-\frac{8}{25} \delta^{\prime}\left(\tau-\sqrt{\frac{3}{5}}|y|\right)\right\}
\end{aligned}
$$

with $\xi_{1}=\sqrt{\tau^{2}-\frac{3}{5} y^{2}}$. It is interesting to note that starting from (48) one can obtain expression (9) corresponding to the exact solution of the kinetic equation in the case $\tau \gg 1$ Eq. (20) has another asymptotic (22).

Quite similarly we can use the diffusion approximation relations and equation for the first harmonic (30) to derive the following modified telegraph equation for $f_{1}$

$$
\frac{1}{5} \frac{\partial^{2} f_{1}}{\partial \tau^{2}}+\frac{\partial f_{1}}{\partial \tau}-\frac{1}{3} \frac{\partial^{2} f_{1}}{\partial y^{2}}=\frac{1}{3 \Lambda}\left\{\frac{4}{5} \delta^{\prime}(y) \delta^{\prime}(\tau)-\delta^{\prime}(y) \delta(\tau)\right\}
$$

The solution of this equation can be written in the form

$$
\begin{aligned}
f_{1}(y, \tau)= & \frac{3 \sqrt{15} y \exp \left(-\frac{5}{2} \tau\right)}{4 \Lambda}\left\{\left[\left(1+\frac{4 \tau}{15 \xi_{1}^{2}}\right) I_{1}\left(\frac{5}{2} \xi_{1}\right)\right.\right. \\
& \left.-\frac{\tau}{3 \xi_{1}}\left[I_{0}\left(\frac{5}{2} \xi_{1}\right)+I_{2}\left(\frac{5}{2} \xi_{1}\right)\right]\right] \frac{\Theta\left(\tau-\sqrt{\frac{3}{5}}|y|\right)}{\xi_{1}} \\
& \left.+\left(\frac{\tau}{y^{2}}-\frac{2}{3}\right) \delta\left(\tau-\sqrt{\frac{3}{5}}|y|\right)-\frac{8}{75 \tau} \delta^{\prime}\left(\tau-\sqrt{\frac{3}{5}}|y|\right)\right\}
\end{aligned}
$$

It is instructive to note that for large values of $\tau$ from (48), (50) we can reach an approximate formula for anisotropy (12). Thus for late times we obtain the same relationship (12) from the exact kinetic equation solution, from the new transport equation and from the modified telegraph equation.

Let us derive the modified telegraph equation corresponding to the Fokker-Planck Eq. (34). In this case from the transport Eq. (37) one can obtain

$$
\begin{aligned}
\frac{11}{15} \frac{\partial^{2} f_{0}}{\partial \tau^{2}}+ & \frac{\partial f_{0}}{\partial \tau}-\frac{1}{3} \frac{\partial^{2} f_{0}}{\partial y^{2}} \\
& =\frac{1}{\Lambda}\left\{\delta(y) \delta(\tau)+\frac{11}{15} \delta(y) \delta^{\prime}(\tau)-\frac{4}{45} \delta^{\prime \prime}(y) \delta(\tau)\right\}
\end{aligned}
$$

This equation differs from (47) by the coefficient at the second order time derivative. As a result, the telegraph Eq. (51) is characterized by the signal propagation speed $v \sqrt{\frac{5}{11}}$, which is 
smaller than the particle velocity. The solution of Eq. (51) can be written as

$$
\begin{aligned}
f_{0}(y, \tau)= & \frac{3 \sqrt{5} \exp \left(-\frac{15}{22} \tau\right)}{4 \sqrt{11} \Lambda}\left\{\left[I_{0}\left(\frac{15}{22} \xi_{2}\right)\right.\right. \\
& +\frac{\tau}{\xi_{2}}\left(1+\frac{4 \tau}{15 \xi_{2}^{2}}\right) I_{1}\left(\frac{15}{22} \xi_{2}\right)-\frac{y^{2}}{5 \xi_{2}^{2}}\left[I_{0}\left(\frac{15}{22} \xi_{2}\right)\right. \\
& \left.+I_{2}\left(\frac{15}{22} \xi_{2}\right)\right] \Theta\left(\tau-\sqrt{\frac{11}{5}}|y|\right) \\
& +2\left(\frac{11}{15}-\frac{\tau}{11}\right) \delta\left(\tau-\sqrt{\frac{11}{5}}|y|\right) \\
& \left.-\frac{88}{225} \delta^{\prime}\left(\tau-\sqrt{\frac{11}{5}}|y|\right)\right\}
\end{aligned}
$$

with $\xi_{2}=\sqrt{\tau^{2}-\frac{11}{5} y^{2}}$. It is instructive to note that in the limit $\tau \gg 1$ from (52) follows the approximate formula for particle density (42), which was derived from solution (38) of transport Eq. (37).

From Eq. (43) one can obtain the following equation for $f_{1}$ $\frac{11}{15} \frac{\partial^{2} f_{1}}{\partial \tau^{2}}+\frac{\partial f_{1}}{\partial \tau}-\frac{1}{3} \frac{\partial^{2} f_{1}}{\partial y^{2}}=\frac{1}{3 \Lambda}\left\{\frac{4}{15} \delta^{\prime}(y) \delta^{\prime}(\tau)-\delta^{\prime}(y) \delta(\tau)\right\}$

which differs from the analogous Eq. (49) only by the coefficient at the second order time derivative and as a consequence incorporates another value of the signal speed. The solution of Eq. (53) has the form

$$
\begin{aligned}
f_{1}(y, \tau)= & \frac{3 \sqrt{5} y \exp \left(-\frac{15}{22} \tau\right)}{4 \sqrt{11} \Lambda}\left\{\left[\left(\frac{13}{11}+\frac{4 \tau}{15 \xi_{2}^{2}}\right)\right.\right. \\
& \left.\times I_{1}\left(\frac{15}{22} \xi_{2}\right)-\frac{\tau}{11 \xi_{2}}\left[I_{0}\left(\frac{15}{22} \xi_{2}\right)+I_{2}\left(\frac{15}{22} \xi_{2}\right)\right]\right] \\
& \times \frac{\Theta\left(\tau-\sqrt{\frac{11}{5}}|y|\right)}{\xi_{2}}+\frac{2}{11}\left(\frac{13}{3} \frac{\tau}{y^{2}}-1\right) \delta\left(\tau-\sqrt{\frac{11}{5}}|y|\right) \\
& \left.-\frac{88}{225 \tau} \delta^{\prime}\left(\tau-\sqrt{\frac{11}{5}}|y|\right)\right\} .
\end{aligned}
$$

Note that under condition $\tau \gg 1$ from (52), (54) there follows an approximate formula for particle anisotropy (46), derived from expressions (41), (44).

\section{Anisotropic scattering}

It is well known that energetic particle scattering in the interplanetary medium is anisotropic (Denskat et al. 1982; Toptygin 1985; Dröge et al. 1993). In the present section we shall derive the CR transport equation taking into account the anisotropic scattering of particles by magnetic irregularities and CR focusing in the diverging interplanetary magnetic field. Energetic particle scattering in the interplanetary medium is caused by magnetic fluctuations, whose frequency spectrum has approximately a power law shape with the spectral index $q$ varying from 1.5 to 1.9 (Denskat et al. 1982; Toptygin 1985; Dröge et al. 1993). The intensity of scattering is characterized by the particle mean free path $\Lambda$, which is associated with a diffusion coefficient in angular space $D_{\mu \mu}$ by a known relation (Hasselmann \& Wibberenz 1968; Beeck \& Wibberenz 1986; Dröge 2000)

$\Lambda=\frac{3 v}{8} \int_{-1}^{1} \mathrm{~d} \mu \frac{\left(1-\mu^{2}\right)^{2}}{D_{\mu \mu}}$

The diffusion coefficient $D_{\mu \mu}$ can be calculated starting from the known spectrum of magnetic field irregularities (Jokipii 1966; Hasselmann \& Wibberenz 1968; Schlickeiser 1989). For a power spectrum with a spectral index $q \neq 1$ the scattering is anisotropic, and the diffusion coefficient can be written in a form (Jokipii 1966; Schlickeiser 1989)

$D_{\mu \mu}=\frac{3 v}{2 \Lambda(2-q)(4-q)}|\mu|^{q-1}\left(1-\mu^{2}\right)$.

Note that under condition $q=1$ isotropic scattering occurs and in this case the quantity $D_{\mu \mu}$ is described by the formula (33).

Energetic particles propagating in the interplanetary medium are focused by the magnetic field owing to decreasing of the field magnitude with heliocentric distance (Earl 1976; Toptygin 1985; Beeck \& Wibberenz 1986). We shall consider this effect in the approximation of the radial regular magnetic field. In this case the focusing length is equal to $\frac{r}{2}$, where $r$ is a heliographic coordinate, and the kinetic equation, describing $\mathrm{CR}$ propagation, takes the form

$\frac{\partial f}{\partial t}+v \mu \frac{\partial f}{\partial r}+\frac{v\left(1-\mu^{2}\right)}{r} \frac{\partial f}{\partial \mu}=S t f+\frac{\delta(r) \delta(t)}{16 \pi^{2} r^{2}}$.

The instantaneous source of particles is included in the right hand side of Eq. (57), and a collision integral $S t f$ represents CR scattering by magnetic irregularities. By neglecting a transfer of magnetic irregularities by the solar wind plasma and an inverse action of CR on the interplanetary magnetic field, the collision integral can be written as

$S t f=2 \pi v n\left\{\int_{-1}^{1} \mathrm{~d} \mu_{1} \sigma\left(\mu, \mu_{1}\right) f\left(\mu_{1}\right)-f(\mu) \int_{-1}^{1} \mathrm{~d} \mu_{1} \sigma\left(\mu, \mu_{1}\right)\right\}$,

where $n$ is the scattering center density, and $\sigma\left(\mu, \mu_{1}\right)$ stands for the scattering cross-section.

In the following we shall assume the scattering to be anisotropic and represent the scattering section as an expansion restricted to the second order Legendre polynomial (Case \& Zweifel 1967)

$\sigma\left(\mu, \mu_{1}\right)=\sigma_{0}\left\{1+3 \alpha \mu \mu_{1}+5 \gamma P_{2}(\mu) P_{2}\left(\mu_{1}\right)\right\}$.

Note that the isotropic scattering is associated with $\alpha=$ $0, \gamma=0$. The scattering is linearly anisotropic if $\gamma=0$, and parameter $\gamma$ characterizes the contribution of quadratic anisotropic scattering (Case \& Zweifel 1967). It is instructive 
to emphasize that the magnitudes of $\alpha$ and $\gamma$ cannot be arbitrary, as the scattering section (59) should acquire only positive values.

Let us define a collision frequency as

$v_{s}=4 \pi v n \sigma_{0}$

and introduce the dimensionless variables

$\rho=\frac{r}{\Lambda}, \tau=\frac{v t}{\Lambda}$,

where $\Lambda=\frac{v}{v_{s}}$ is the particle mean free path. Then the kinetic Eqs. (57), (58) can be rewritten in the form

$$
\begin{aligned}
\frac{\partial f}{\partial \tau}+\mu \frac{\partial f}{\partial \rho}+ & \frac{1-\mu^{2}}{\rho} \frac{\partial f}{\partial \mu}+f-\frac{1}{4 \pi} f_{0} \\
& -\frac{3 \alpha}{4 \pi} \mu f_{1}-\frac{5 \gamma}{4 \pi} P_{2}(\mu) f_{2}=\frac{\delta(\rho) \delta(\tau)}{16 \pi^{2} \Lambda^{3} \rho^{2}},
\end{aligned}
$$

where the relationship

$f_{n}(\rho, \tau)=2 \pi \int_{-1}^{1} \mathrm{~d} \mu P_{n}(\mu) f(\rho, \tau, \mu)$

defines the harmonics of the CR distribution function.

Multiplying Eq. (62) accordingly by $1, \mu$ and $P_{2}(\mu)$ and integrating over $\mu$ from -1 to 1 , one can obtain a set of equations for distribution function harmonics

$\frac{\partial f_{0}}{\partial \tau}+\frac{1}{\rho^{2}} \frac{\partial}{\partial \rho} \rho^{2} f_{1}=\frac{\delta(\rho) \delta(\tau)}{4 \pi \Lambda^{3} \rho^{2}} ;$

$\frac{\partial f_{1}}{\partial \tau}+(1-\alpha) f_{1}+\frac{1}{3} \frac{\partial f_{0}}{\partial \rho}+\frac{2}{3} \frac{\partial f_{2}}{\partial \rho}+\frac{2}{\rho} f_{2}=0 ;$

$\frac{\partial f_{2}}{\partial \tau}+(1-\gamma) f_{2}+\frac{2}{5} \frac{\partial f_{1}}{\partial \rho}+\frac{3}{5} \frac{\partial f_{3}}{\partial \rho}-\frac{2}{5 \rho} f_{1}+\frac{12}{5 \rho} f_{3}=0$.

Neglecting quantities $f_{2}$ and $\frac{\partial f_{1}}{\partial \tau}$ in (65) one can derive from (64), (65) the diffusion equation. It should be noted that the derived equation is associated with a diffusion coefficient which differs from the corresponding value under isotropic scattering by the $(1-\alpha)^{-1}$ multiplier.

We neglect in (66) the third harmonic and the time derivative, and we can obtain the approximate relationship for the second harmonic of the $\mathrm{CR}$ distribution function

$f_{2}=-\frac{2}{5(1-\gamma)} \rho \frac{\partial}{\partial \rho} \frac{f_{1}}{\rho}$.

Using (67), from Eqs. (64), (65), we get the following transport equation

$$
\begin{aligned}
\frac{\partial^{2} f_{0}}{\partial \tau^{2}}+ & (1-\alpha) \frac{\partial f_{0}}{\partial \tau}-\frac{1}{3 \rho^{2}} \frac{\partial}{\partial \rho} \rho^{2} \frac{\partial f_{0}}{\partial \rho} \\
& -\frac{4}{15(1-\gamma) \rho^{2}} \frac{\partial}{\partial \rho} \rho^{2} \frac{\partial^{2} f_{0}}{\partial \rho \partial \tau}=\frac{1}{4 \pi \Lambda^{3} \rho^{2}}\{(1-\alpha) \delta(\rho) \delta(\tau) \\
& \left.+\delta(y) \delta^{\prime}(\tau)-\frac{4}{15(1-\gamma)} \delta(\tau) \frac{\partial}{\partial \rho} \rho^{2} \frac{\partial}{\partial \rho} \frac{\delta(\rho)}{\rho^{2}}\right\}
\end{aligned}
$$

The solution of Eq. (68) can be reached by integral transformations (Appendix C) and has the following form

$$
\begin{aligned}
f_{0}(y, \tau)= & \frac{\exp \left(-\frac{(1-\alpha) \tau}{2}\right)}{2 \pi^{2} \Lambda^{3} \rho} \int_{0}^{\infty} \mathrm{d} k k \sin k \rho \\
& \times \exp \left(-\frac{2}{15(1-\gamma)} k^{2} \tau\right)\left\{\left[k^{2}+\frac{15}{4}(1-\alpha)(1-\gamma)\right]\right. \\
& \times \frac{1}{\sqrt{\Gamma}} \sinh \left(\frac{2}{15(1-\gamma)} \tau \sqrt{\Gamma}\right) \\
& \left.+\cosh \left(\frac{2}{15(1-\gamma)} \tau \sqrt{\Gamma}\right)\right\}
\end{aligned}
$$

with

$$
\begin{aligned}
\Gamma(k)= & k^{4}-\frac{15}{4}(1-\gamma)(3+2 \alpha-5 \gamma) k^{2} \\
& +\left[\frac{15}{4}(1-\alpha)(1-\gamma)\right]^{2}
\end{aligned}
$$

It can be shown (Appendix A) that long after injection (i.e. at large values of dimensionless time $\tau$ ) the expression (69) simplifies essentially

$$
\begin{aligned}
f_{0}(y, \tau)= & \frac{[3(1-\alpha)]^{\frac{3}{2}} \exp \left(-\frac{3 \rho^{2}(1-\alpha)}{4 \tau}\right)}{8 \pi^{\frac{3}{2}} \Lambda^{3} \tau^{\frac{3}{2}}} \\
& \times\left\{1+\frac{3}{4} \frac{1-4 \alpha+3 \gamma}{(1-\alpha)(1-\gamma) \tau}+3 \frac{(\alpha-\gamma) \rho^{2}}{(1-\gamma) \tau^{2}}\right\} .
\end{aligned}
$$

It should be noted that the solution of the diffusion equation follows from (71) by neglecting two last terms in brackets. It is obvious from (71) that the linearly anisotropic scattering results in a mean free path modification, thus the multiplier $(1-\alpha)^{-1}$ appears in the mean free path expression in comparison with the isotropic scattering case. The calculations demonstrate that solution (69) of the transport Eq. (68) corresponds to the later onset of CR intensity enhancement and to the sharper maximum of a temporal intensity profile compared to the solution of the diffusion equation. The value of the maximum intensity enhancement and the sharpness of time intensity profile rises with increasing of $\alpha$, describing the contribution of linearly anisotropic scattering. The increase of quadratic anisotropic scattering (represented by $\gamma$ ) leads to a more smoothly varying temporal intensity profile and to a decrease of maximum value of the SCR enhancement.

In order to illustrate the temporal profile of SCR enhancement, described by the relationship (69), we have made some calculations concerning the solar proton event of July 14, 2000. This SCR flare was most powerful in the current cycle of solar activity until April, 2001 (Belov et al. 2001). The anisotropy data of relativistic protons, obtained on a world-wide network of neutron monitors, indicate the impulsive nature of this event (Belov et al. 2001; Pchelkin et al. 2001; Bieber et al. 2002), that allows us to use the solution of the transport equation associated with an instantaneous particle injection. We shall consider 10.20 UT as the moment of energetic particle release from the Sun of July 14, 2000; this time is associated with the start of radiowave, X-ray and $\gamma$-ray emission (Belov et al. 2001; Pchelkin et al. 2001; Bieber et al. 2002). It should be emphasized that the 


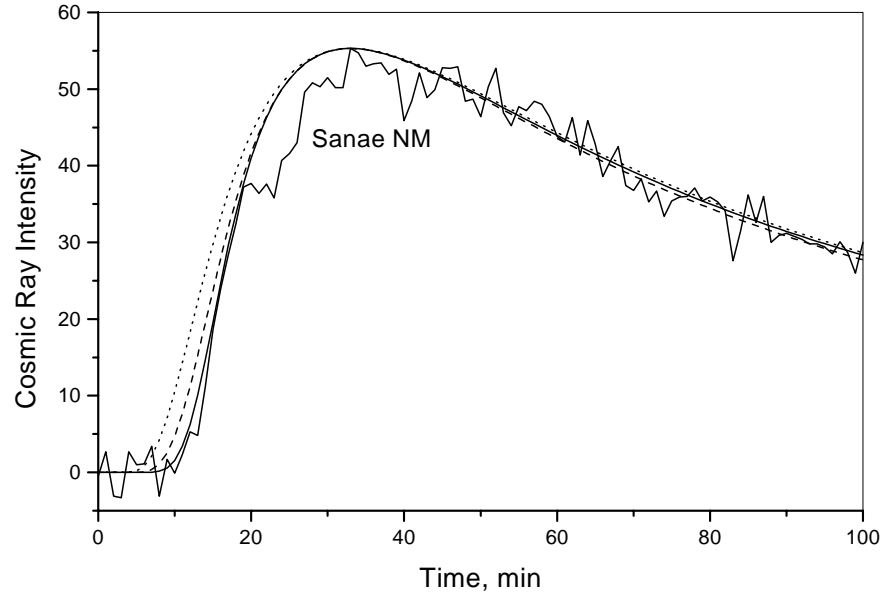

Fig. 5. The temporal profile of solar particle event of July 14, 2000, as recorded by the neutron monitor Sanae and calculated enhancements of CR intensity. Time is in minutes after energetic particle injection near the Sun (the SCR release was supposed to occur at 10.20 UT). The dotted curve corresponds to the diffusion approximation, the dashed curve illustrates the solution (69) under isotropic scattering, and the solid line is associated with (69) in the case of linearly anisotropic scattering $(\alpha=1 / 3, \gamma=0)$.

arrival of the first relativistic protons was registered on Earth at 10.30 UT which does not contradict a particle injection near the Sun at $10.20 \mathrm{UT}$.

In Fig. 5 the 1-min data of the neutron monitor Sanae are shown; this CR station detected one of the most considerable enhancements the neutron monitors network in a given event (maximum intensity enhancement according to 1-min data amounts to 53 percent relative to the galactic CR background). The time in minutes is given in Fig. 5 after the injection moment at 10.20 . It is instructive to note the complex shape of the experimental curve on the rising phase of CR intensity, starting around the 20th minute and lasting about $20 \mathrm{~min}$. Possible reasons for this effect could be inhomogeneous local propagation conditions of energetic particles or fluctuations of the interplanetary magnetic field orientation. The dotted curve in Fig. 5 corresponds to the diffusion equation solution, the dashed curve represents the solution (69) of the transport Eq. (68) under isotropic scattering, and the solid curve introduces the case of linearly anisotropic scattering $(\gamma=0, \alpha=1 / 3)$. In all cases the mean free path was selected from a condition of coincidence of the calculated CR maximum with the moment of maximal intensity detected on the neutron monitor Sanae (33 min after 10.20 UT of July 14, 2000). The mean free path $\Lambda=0.17$ AU corresponds to isotropic scattering, and $\Lambda=0.11 \mathrm{AU}$ is associated with the linearly anisotropic scattering according to a decrease in $\Lambda$ with increase of scattering anisotropy.

The event of July 14, 2000 is characterized by sufficiently intensive scattering, so the mean free path is small in comparison to the distance to the particle source. Because of this the relationship (69) (the dashed and solid curves in Fig. 5) differ markedly from the solution of the diffusive equation (dotted curve) only during about the first $15 \mathrm{~min}$ after the first particle arrival. The temporary profiles of SCR intensity, calculated according to (69) (solid and dashed curves in Fig. 5) are different from one another for a still shorter period of time. Note that the calculation in the case of anisotropic scattering (solid curve in Fig. 5) better approximates the early phase of CR intensity enhancement compared to the isotropic scattering (dashed curve in Fig. 5).

\section{Summary}

In the present paper the propagation of charged energetic particles in homogeneous regular magnetic field under isotropic scattering on magnetic irregularities is considered. The exact analytical solutions of the Boltzmann equation for particle density and anisotropy are derived and these solutions are compared with results obtained with various approximate methods (such as diffusion approximation, telegraph equation description and the new transport equation approach). It is shown that the obtained expressions (9), (12) (valid for late times) in a zero order approximation with respect to $\frac{1}{\tau}$ coincide with the diffusion and telegraph equation descriptions. It is worth noting that in the first order approximation relative to $\frac{1}{\tau}$ the results obtained from the kinetic equation and from the telegraph equation approach differ from one another.

The new CR transport equations taking into account the second harmonic of the distribution function are derived and solutions of these equations are presented. The calculations show that density and anisotropy values obtained from the new transport equation fit the exact solutions of the Boltzmann equation much better than corresponding expressions derived with diffusion approximation or with the telegraph equation approach. At late times the solutions of the new transport equations satisfy the relationships (9), (12), obtained from kinetic equation solutions. It is instructive to note that solutions of the new transport Eqs. (28), (31) describe particle distribution with good accuracy but not shortly after injection $\left(t>\frac{\Lambda}{v}\right)$ for reasonably short distances from the particle source $(z<v t)$, it is not close to the boundaries of the region filled by particles.

The CR propagation also can be studied on the basis of the Fokker-Planck equation, describing the process of multiple small angle scattering. The diffusion and telegraph equations, derived from the Fokker-Planck equation, are the same as from the Boltzmann equation, provided the mean free path values coincide. However, the new transport Eq. (37), derived from the Fokker-Planck equation, is different from the corresponding Eq. (27). Calculations show that in the case of multiple small angle scattering the temporal profile of particle density exhibit a sharper maximum and anisotropy takes higher values in comparison with corresponding quantities, obtained on the basis of the Boltzmann equation.

Starting from kinetic Eqs. (1) and (32), modified telegraph equations for particle density and anisotropy are obtained and solutions of these equations are presented. Derived Eqs. (47), (51) correspond to different speeds of disturbance propagation in accordance with conclusions of Gombosi et al. (1993). It is interesting to note that at late times the results for particle density and anisotropy obtained from new transport equations and derived from modified telegraph equations coincide in the first order approximation with respect to the small parameter $\frac{1}{\tau}$. 
The new transport equation derived in this paper allows us to describe the spatial-temporal particle distribution with good accuracy if the time after injection is not small in comparison with the inverse scattering frequency. It is instructive to note that this description proves to be more correct than the diffusion and telegraph equation approximations. This approach can be successfully applied to problems more complicated than the process of $\mathrm{CR}$ propagation in homogeneous regular magnetic fields under isotropic scattering of particles, considered in this paper.

On the basis of a kinetic equation taking into account magnetic focusing of energetic charged particles and the anisotropic nature of CR scattering on magnetic irregularities, a new transport equation is derived. The regular magnetic field was supposed to be radial, and the particle scattering cross-section on "magnetic clouds" was described by relationship (59) (Case $\&$ Zweifel 1967). The solution of this equation is applied to the analysis of Sanae neutron monitor data for the solar proton event of July 14, 2000. It is shown that for this event the case of linearly anisotropic scattering better approximates the initial phase of SCR enhancement than the isotropic scattering approach.

\section{Appendix A}

In this appendix we shall consider briefly the derivation of expressions for particle density $f_{0}$ and for the first harmonic of the distribution function $f_{1}$ starting from the kinetic equation. We shall deduce also the approximate formulas (9), (12) which are valid for late times after particle injection.

Let us introduce a new function according to the following equation

$\Phi(y, \tau, \mu)=\exp (\tau) f(y, \tau, \mu)$.

By performing a Fourier transform of the spatial variable $y$ and a Laplace transform of the temporal variable $\tau$

$\Phi(k, \omega, \mu)=\int_{-\infty}^{\infty} \mathrm{d} y \int_{0}^{\infty} \mathrm{d} \tau \exp (-\mathrm{i} k y-\omega \tau) \Phi(y, \tau, \mu)$

we can derive from kinetic Eq. (2) the following formula for the Fourier-Laplace transform of the distribution function

$\Phi(k, \omega, \mu)=\frac{1}{2 \Lambda(\omega+\mathrm{i} k \mu)}+\frac{\arctan \frac{k}{\omega}}{2 \Lambda(\omega+\mathrm{i} k \mu)\left(k-\arctan \frac{k}{\omega}\right)}$.

The first term in formula (A.3) corresponds to unscattered particles, whose distribution function can be obtained by performing the inverse Laplace and Fourier transformations. In order to derive the Fourier-Laplace transform of scattered particle density we integrate the second term in (A.3) over $\mu$

$\Phi_{0}^{\mathrm{s}}(k, \omega)=\frac{\arctan ^{2} \frac{k}{\omega}}{\Lambda k\left(k-\arctan \frac{k}{\omega}\right)}$.

The expression for a Fourier-Laplace transform of the first harmonic of the scattered distribution function can be obtained by multiplying the second term in (A.3) by $\mu$ and by integrating over $\mu$ from -1 to 1

$\Phi_{1}^{\mathrm{s}}(k, \omega)=-\frac{i \arctan \frac{k}{\omega}\left(k-\omega \arctan \frac{k}{\omega}\right)}{\Lambda k^{2}\left(k-\arctan \frac{k}{\omega}\right)}$.

Following from our previous papers (Fedorov \& Shakhov 1994; Fedorov et al. 1995) we perform first an inverse Laplace transformation. The function of the complex variable $\omega$

$\arctan \frac{k}{\omega}=\frac{1}{2 \mathrm{i}} \ln \frac{\omega+\mathrm{i} k}{\omega-\mathrm{i} k}$

has two branch points $\omega= \pm \mathrm{i} k$ (Sidorov et al. 1982), therefore to define unique branches of the analytic functions (A.4), (A.5) it is necessary to make a branch cut in the complex $\omega$-plane along the imaginary axis from $-\mathrm{i} k$ to $\mathrm{i} k$. The equation

$k-\arctan \frac{k}{\omega}=0$

has a unique root

$\omega_{0}=k \cot k$

if the condition $-\frac{\pi}{2}<k<\frac{\pi}{2}$ is satisfied. In the opposite case $|k|>\frac{\pi}{2}$ the Eq. (A.7) has no solution. Therefore, functions (A.4), (A.5) of the complex variable $\omega$ have two branch points $\omega= \pm \mathrm{i} k$ and the pole of the first order $\omega_{0}$ (A.8). The inverse Laplace transform leads to the following expressions:

$$
\begin{aligned}
\Phi_{0}^{\mathrm{s}}(k, \tau)= & \frac{k^{2} \exp (\tau k \cot k)}{\Lambda \sin ^{2} k}\left\{\Theta\left(k+\frac{\pi}{2}\right)\right. \\
& \left.-\Theta\left(k-\frac{\pi}{2}\right)\right\}+\frac{1}{8 \pi \Lambda} \int_{-1}^{1} \mathrm{~d} \eta\left[\frac{(\pi+\mathrm{i} \kappa)^{2}}{k-\frac{\pi}{2}-\frac{\mathrm{i}}{2} \kappa}\right. \\
& \left.-\frac{(\pi-\mathrm{i} \kappa)^{2}}{k+\frac{\pi}{2}-\frac{\mathrm{i}}{2} \kappa}\right] \exp (\mathrm{i} k \tau \eta),
\end{aligned}
$$

$$
\begin{aligned}
\Phi_{1}^{\mathrm{s}}(k, \tau)= & -\frac{\mathrm{i} k(\sin k-k \cos k) \exp (\tau k \cot k)}{\Lambda \sin ^{2} k} \\
& \times\left\{\Theta\left(k+\frac{\pi}{2}\right)-\Theta\left(k-\frac{\pi}{2}\right)\right\} \\
& -\frac{\mathrm{i}}{8 \pi \Lambda} \int_{-1}^{1} \mathrm{~d} \eta\left[\frac{(\pi-\mathrm{i} \kappa)\left(2+\mathrm{i} \eta \pi+\eta \kappa^{2}\right)}{k+\frac{\pi}{2}-\frac{\mathrm{i}}{2} \kappa}\right. \\
& \left.+\frac{(\pi+\mathrm{i} \kappa)\left(2-\mathrm{i} \eta \pi+\eta \kappa^{2}\right)}{k-\frac{\pi}{2}-\frac{\mathrm{i}}{2} \kappa}\right] \exp (\mathrm{i} k \tau \eta),
\end{aligned}
$$

with $\kappa=\ln \frac{1-\eta}{1+\eta}$. The first term in formulas (A.9), (A.10) is due to the calculation of the pole in the point $\omega_{0}$ (A.8) and the condition of this singularity existence is manifested as the difference of the Heaviside step functions. The second term in (A.9), (A.10) arises as a result of integration along the edges of the branch line on the imaginary axis. Performing the inverse Fourier transformation we obtain expressions (8) and (10), describing two first harmonics of the scattered distribution function.

Let us now consider the approximate formulas valid long after injection when particles have undergone many scatters and the distribution function becomes close to isotropic. In this 
case we can neglect the second term in the formula for scattered density (8), which is proportional to the exponentially small multiplier. If the time after particle release is much more than the scattering time, the number of unscattered particles (7) is also negligible. The integrand in the first term in (8) has a sharp maximum in the neighborhood of $k=0$. Thus, expanding the integrand in a series near $k=0$ and extending the integration to the infinity yields

$f_{0}(y, \tau)=\frac{1}{\pi \Lambda} \int_{0}^{\infty} \mathrm{d} k \cos k y \exp \left(-\frac{k^{2} \tau}{3}\right)\left\{1+\frac{k^{2}}{3}-\frac{k^{4}}{45} \tau\right\}$.

Then we can integrate (A.11) using known relationships (Prudnikov et al. 1981) and obtain expression (9) for the particle density, which is valid under the condition $\tau \gg 1$.

From relationship (10), describing the first harmonic of scattered particle distribution, one can obtain the expression valid for late times

$f_{1}(y, \tau)=\frac{1}{3 \pi \Lambda} \int_{0}^{\infty} \mathrm{d} k k \sin k y \exp \left(-\frac{k^{2} \tau}{3}\right)\left\{1+\frac{2 k^{2}}{5}-\frac{k^{4}}{45} \tau\right\}$.

After integration (Prudnikov et al. 1981) we derive the following expression

$f_{1}(y, \tau)=\frac{\sqrt{3} y \exp \left(-\frac{3 y^{2}}{4 \tau}\right)}{4 \Lambda \sqrt{\pi} \tau^{3 / 2}}\left\{1+\frac{21}{20 \tau}-\frac{3 y^{2}}{20 \tau^{2}}\right\}$.

Taking into account the minority of quantities $\frac{1}{\tau}$ and $\frac{y^{2}}{\tau^{2}}$ from Eqs. (A.13), (9) one can obtain a formula for particle anisotropy (12).

\section{Appendix B}

We present the derivation of solution (21) of the telegraph Eq. (20). If we introduce a new function

$\Phi_{0}(y, \tau)=f_{0}(y, \tau) \exp \left(\frac{\tau}{2}\right)$

Equation (20) takes the form

$$
\begin{aligned}
\frac{\partial^{2} \Phi_{0}}{\partial \tau^{2}}- & \frac{1}{4} \Phi_{0}-\frac{1}{3} \frac{\partial^{2} \Phi_{0}}{\partial y^{2}} \\
& =\frac{1}{\Lambda}\left\{\delta(y) \delta(\tau)+\delta(y) \delta^{\prime}(\tau)\right\} \exp \left(\frac{\tau}{2}\right)
\end{aligned}
$$

From (B.2) using (A.2) one can reach the following formula for the Fourier-Laplace transform of function $\Phi_{0}$

$\Phi_{0}(k, \omega)=\frac{1}{\Lambda} \frac{\omega+\frac{1}{2}}{\omega^{2}-\frac{1}{4}+\frac{k^{2}}{3}}$.

Performing an inverse Fourier transformation we obtain

$\Phi_{0}(y, \omega)=\frac{\left.\sqrt{3(} \omega+\frac{1}{2}\right)}{2 \Lambda} \frac{\exp \left(-\sqrt{3}|y| \sqrt{\omega^{2}-\frac{1}{4}}\right)}{\sqrt{\omega^{2}-\frac{1}{4}}}$.
In order to perform an inverse Laplace transformation one can use the following relationship (Sidorov et al. 1982)

$$
\begin{array}{r}
\frac{1}{2 \pi i} \int_{L} \mathrm{~d} \omega \exp (\omega \tau) \frac{\exp \left(-\sqrt{3}|y| \sqrt{\omega^{2}-\frac{1}{4}}\right)}{\sqrt{\omega^{2}-\frac{1}{4}}} \\
=I_{0}\left(\frac{1}{2} \sqrt{\tau^{2}-3 y^{2}}\right) \Theta(\tau-\sqrt{3}|y|) .
\end{array}
$$

Thus one can obtain the expression

$\Phi_{0}(y, \tau)=\frac{\sqrt{3}}{4 \Lambda}\left(1+2 \frac{\partial}{\partial \tau}\right) I_{0}\left(\frac{1}{2} \sqrt{\tau^{2}-3 y^{2}}\right) \Theta(\tau-\sqrt{3}|y|)$,

which is followed by formula (21) for particle density.

It is worth noting that expression (22) valid under condition $\tau \gg 1$ can be obtained from (21) by applying an asymptotic expression of a modified Bessel function, which is valid for large values of $x$ (Abramowitz \& Stegun 1964)

$I_{n}(x) \simeq \frac{\exp (x)}{\sqrt{2 \pi x}}\left(1-\frac{4 n^{2}-1}{8 x}\right)$.

\section{Appendix C}

In this section we present the derivation of solution (28) of the transport Eq. (27). Let us introduce the function $\Phi_{0}$ according to (B.1). Performing the Fourier-Laplace transformation of Eq. (27) one can obtain the following formula

$$
\begin{aligned}
\Phi_{0}(k, \omega)= & \frac{15}{4 \Lambda} \frac{\omega+\frac{4}{15} k^{2}+\frac{1}{2}}{\sqrt{\Psi}} \\
& \times\left\{\frac{1}{\omega+\frac{2}{15} k^{2}+\sqrt{\Psi}}-\frac{1}{\omega+\frac{2}{15} k^{2}-\sqrt{\Psi}}\right\},
\end{aligned}
$$

with $\Psi$ defined by (29).

Let us perform first an inverse Laplace transformation. The function of the complex variable $\omega$ (C.1) has two poles, so by calculating the residual values we can obtain the relationship

$$
\begin{aligned}
\Phi_{0}(k, \tau)= & \frac{\exp \left(-\frac{2}{15} k^{2} \tau\right)}{\Lambda}\left\{\left(k^{2}+\frac{15}{4}\right) \frac{\sinh \left(\frac{2}{15} \tau \sqrt{\Psi}\right)}{\sqrt{\Psi}}\right. \\
& \left.+\cosh \left(\frac{2}{15} \tau \sqrt{\Psi}\right)\right\}
\end{aligned}
$$

Performing an inverse Fourier transformation of (C.2) we derive expression (28) for particle density.

Note that the function $\Psi(k)$ (29) becomes negative for $k_{1}<$ $k<k_{2}$, where

$k_{1}^{2}=\frac{15}{8}(3-\sqrt{5}) ; k_{2}^{2}=\frac{15}{8}(3+\sqrt{5})$.

Thus, the interval of integration in (28) splits into three parts, with the integrand as in (28) under integration from 0 to $k_{1}$ and from $k_{2}$ to the infinity, while if $k_{1}<k<k_{2}$ it is necessary in (28) to replace $\sinh (x)$ and $\cosh (x)$ by $\sin (x)$ and $\cos (x)$ respectively. Consequently, we can rewrite (28) as

$f_{0}(y, \tau)=f_{0}^{\alpha}(y, \tau)+f_{0}^{\beta}(y, \tau)+f_{0}^{\gamma}(y, \tau)$, 
with

$$
\begin{aligned}
f_{0}^{\beta}(y, \tau)= & \frac{\exp \left(-\frac{\tau}{2}\right)}{\pi \Lambda} \int_{k_{1}}^{k_{2}} \mathrm{~d} k \cos k y \exp \left(-\frac{2}{15} k^{2} \tau\right) \\
& \times\left\{\left(k^{2}+\frac{15}{4}\right) \frac{\sin \left(\frac{2}{15} \tau \sqrt{-\Psi}\right)}{\sqrt{-\Psi}}+\cos \left(\frac{2}{15} \tau \sqrt{-\Psi}\right)\right\},
\end{aligned}
$$

and $f_{0}^{\alpha}, f_{0}^{\gamma}$ differ from (28) only by the limits of integration.

One can show that for large values of $\mathrm{k}$ the integrand in (28) approaches $\exp \left(-\frac{3 \tau}{4}\right) \cos k y$. Let us rewrite expression (28) in the form

$$
\begin{aligned}
f_{0}(y, \tau)= & \frac{\exp \left(-\frac{\tau}{2}\right)}{\pi \Lambda} \int_{0}^{\infty} \mathrm{d} k \cos k y\left\{\exp \left(-\frac{2}{15} k^{2} \tau\right)\right. \\
& \times\left[\left(k^{2}+\frac{15}{4}\right) \frac{\sinh \left(\frac{2}{15} \tau \sqrt{\Psi}\right)}{\sqrt{\Psi}}+\cosh \left(\frac{2}{15} \tau \sqrt{\Psi}\right)\right] \\
& \left.-\exp \left(-\frac{3}{4} \tau\right)\right\}+\frac{\exp \left(-\frac{5}{4} \tau\right)}{\Lambda} \delta(y) .
\end{aligned}
$$

Note that from (C.6) the singularity occurrence in $y=0$ is evident. The integrand in (C.6) decays with increasing $k$ proportional to $\frac{\cos k y}{k^{2}}$, so the integral in (C.6) converges quite well (in contrast with expression (C.5)). The singularity occurrence in (C.6) is due to the approximation used in the transport equation derivation, i.e. results from replacement of (15) by the approximate formula (26).

Quite similarly one can show that the integrand in Eq. (31), describing the first harmonic of the particle distribution, approaches at large $k$ to $\exp \left(-\frac{3 \tau}{4}\right) \frac{\sin k y}{2 k}$. Thus, the relationship (31) can be rewritten as

$$
\begin{aligned}
f_{1}(y, \tau)= & \frac{5 \exp \left(-\frac{\tau}{2}\right)}{2 \pi \Lambda} \int_{0}^{\infty} \mathrm{d} k k \sin k y \\
& \times\left\{\exp \left(-\frac{2}{15} k^{2} \tau\right) \frac{\sinh \left(\frac{2}{15} \tau \sqrt{\Psi}\right)}{\sqrt{\Psi}}-\frac{\exp \left(-\frac{3}{4} \tau\right)}{2 k^{2}}\right\} \\
& +\frac{5 \exp \left(-\frac{5}{4} \tau\right)}{4 \Lambda}\left\{\Theta(y)-\frac{1}{2}\right\}
\end{aligned}
$$

From (C.7) the jump of $f_{1}$ in the $y=0$ position is evident. Note that the magnitude of this jump decays exponentially with time. The occurrence of such a discontinuity in $f_{1}$ at the origin is typical only of a given approximation. In fact the quantity $f_{1}(7),(10)$ derived from the kinetic equation approaches zero continuously when $y$ tends to 0 .

\section{References}

Abramowitz, M., \& Stegun, I. A. 1964, Handbook of mathematical functions (New York: Dover), 832
Achatz, U., Steinacker, J., \& Schlickeiser, R. 1991, A\&A, 250, 266

Bazilevskaya, G. A., \& Golynskaya, R. M. 1989, Geomagn. Aeronomy, 29, 204

Beeck, J., \& Wibberenz, G. 1986, ApJ, 311, 437

Belov, A. V., Bieber, J. W., Eroshenko, E. A., et al. 2001, Proc. 27th Intern. Cosmic Ray Conf. (Hamburg), 3, 3446

Bieber, J. W., Dröge, W., Evenson, P. A., et al. 2002, ApJ, 567, 622

Case, K. M., \& Zweifel, P. F. 1967, Linear transport theory (Reading: Addison-Wesley Publ. Comp.)

Chuvilgin, L. G., \& Ptuskin, V. S. 1993, A\&A, 279, 278

Denskat, K. U., \& Neubauer, F. M. 1982, JGR, 87, 2215

Dolginov, A. Z., \& Toptygin, I. N. 1967, Geomagn. Aeronomy, 7, 967

Dorman, L. I., \& Katz, M. E. 1977, Space Sci. Rev., 20, 529

Dorman, L. I., Katz, M. E., \& Stehlik, M. 1990, Bull. Astron. Inst. Czecosl., 41, 312

Dorman, L. I., Katz, M. E., Fedorov, Yu. I., \& Shakhov, B. A. 1983, Proc. 18th Intern. Cosmic Ray Conf. (Bangalore), 10, 63

Dröge, W., Achatz, U., Wanner, W., Schlickeiser, R., \& Wibberenz, G. 1993, ApJ, 407, L95

Dröge, W. 2000, ApJ, 537, 1073

Earl, J. A. 1973, ApJ, 180, 227

Earl, J. A. 1976, ApJ, 205, 900

Earl, J. A. 1984, ApJ, 278, 825

Earl, J. A. 1994, ApJ, 425, 331

Fedorov, Yu. I., Katz, M. E., Kichatinov, L. L., \& Stehlik, M. 1992, A\&A, 260, 499

Fedorov, Yu. I., \& Shakhov, B. A. 1993, Proc. 23rd Intern. Cosmic Ray Conf. (Calgary), 3, 215

Fedorov, Yu. I., \& Shakhov, B. A. 1994, Geomagn. Aeronomy, 34, 19 Fedorov, Yu. I., Shakhov, B. A., \& Stehlik, M. 1995, A\&A, 302, 623

Fisk, L. A., \& Axford, W. I. 1969, Sol. Phys., 7, 486

Gleeson, L. J., \& Axford, W. I. 1967, ApJ, 149, L115

Gombosi, T. J., Jokipii, J. R., Kota, J., Lorencz, K., \& Williams, L. L. 1993, ApJ, 403, 377

Hasselmann, K., \& Wibberenz, G. 1968, Z. Geophys., 34, 353

Jokipii, J. R. 1966, ApJ, 146, 480

Kirk, J. G., Duffy, P., \& Gallant, Y. A. 1996, A\&A, 314, 1010

Kota, J. 1994, ApJ, 427, 1035

Michalek, G., \& Ostrowski, M. 1997, A\&A, 326, 793

Pchelkin, V. V., Vashenyuk, E. V., \& Gvozdevsky, B. B. 2001, Proc. 27th Intern. Cosmic Ray Conf. (Hamburg), 3, 3379

Prudnikov, A. P., Brychkov, Yu. A., \& Marichev, O. I. 1981, Integrals and series (Moscow: Nauka), 800

Schlickeiser, R. 1989, ApJ, 336, 243

Schlickeiser, R., Dung, R., \& Jackel, U. 1991, A\&A, 242, L5

Shishov, V. I. 1966, Geomagn. Aeronomy, 6, 223

Sidorov, Yu. V., Fedoryuk, M. V., \& Shabunin, M. I. 1982, The lectures on the theory of complexe variable functions (Moscow: Nauka), 488

Toptygin, I. N. 1985, Cosmic rays in interplanetary magnetic fields (Dordrecht: Reidel), 302

Webb, G. M., Pantazopoulou, M., \& Zank, G. P. 2000, J. Phys. A. Math. Gen., 33, 3137

Zank, G. P., Lu, J. Y., Rice, W. K. M., \& Webb, G. M. 1999, Proc. 26th Intern. Cosmic Ray Conf. (Salt Lake City), 6, 312 